\title{
A Predator's Perspective of the Accuracy of Ant Mimicry in Spiders
}

\author{
Ximena J. Nelson \\ School of Biological Sciences, University of Canterbury, Private Bag 4800, Christchurch 8041, New Zealand \\ Correspondence should be addressed to Ximena J. Nelson, ximena.nelson@canterbury.ac.nz
}

Received 28 April 2011; Accepted 9 June 2011

Academic Editor: Jean Paul Lachaud

Copyright () 2012 Ximena J. Nelson. This is an open access article distributed under the Creative Commons Attribution License, which permits unrestricted use, distribution, and reproduction in any medium, provided the original work is properly cited.

\begin{abstract}
Among spiders, resemblance of ants (myrmecomorphy) usually involves the Batesian mimicry, in which the spider coopts the morphological and behavioural characteristics of ants to deceive ant-averse predators. Nevertheless, the degree of resemblance between mimics and ants varies considerably. I used Portia fimbriata, a jumping spider (Salticidae) with exceptional eyesight that specialises on preying on salticids, to test predator perception of the accuracy of ant mimicry. Portia fimbriata's response to ants (Oecophylla smaragdina), accurate ant-like salticids (Synageles occidentalis), and inaccurate ant-like salticids (females of Myrmarachne bakeri and sexually dimorphic males of M. bakeri, which have enlarged chelicerae) was assessed. Portia fimbriata exhibited graded aversion in accordance with the accuracy of resemblance to ants (O. smaragdina $>$ S. occidentalis $>$ female M. bakeri $>$ male M. bakeri). These results support the hypothesis that ant resemblance confers protection from visual predators, but to varying degrees depending on signal accuracy.
\end{abstract}

\section{Introduction}

Predator avoidance of dangerous prey is often exploited by deceptive prey species; the Batesian mimics are those that deceitfully advertise to potential predators that they also can induce the negative repercussions associated with this prey $[1,2]$, which often use warning (aposematic) signals to indicate their defences to would-be predators. The Batesian mimicry works solely to the advantage of the sender of the counterfeit signal, as both the receiver and the model are exploited. The receiver is cheated out of a source of food, and the model is less likely to benefit from its cues. The negative effect on models is due to frequency-dependent selection: if mimics exist in large numbers, the predators may take longer to learn an aversion or the potential for evolving innate fear of dangerous prey is lessened. Although studies of the Batesian mimicry have usually emphasised learning as a mechanism for the evolution of mimicry (e.g., [3]), both innate and learned fear of dangerous or distasteful prey can favour the evolution of the Batesian mimicry, as is clear from studies using naïve jumping spiders (Salticidae) as potential predators (e.g., [4]).
While we traditionally think of dangerous prey as one using bright, contrasting colours as aposematic signals, as in the case of poison dart frogs [3], not all dangerous species that are mimicked use aposematic signals. Correspondingly, deceitful use of aposematic signals appears to be an evolutionary strategy used by some Batesian mimics, but not others. Many spiders are the Batesian mimics of ants [5], animals which do not intuitively fit into the category of aposematic. Having a slender body, narrow waist, and an erratic style of locomotion, ants have a distinctive appearance, but this is unlikely to have evolved as an antipredator defence signal. Ants are, nevertheless, potentially harmful to predators through their ability to bite, sting, or spray formic acid. Being social, ants are all the more dangerous because they can mount communal attacks on potential predators [6]. Predators often respond to ant-like appearance as a cue for avoidance [4], and to disqualify ant mimicry as examples of the Batesian mimicry on the basis of hypotheses about the evolutionary origin of the ant's appearance places undue emphasis on a distinction that is irrelevant to the predator. In fact, ants appear to be particularly suitable as models for mimicry, especially among spiders. Illustrating how 
predation plays an important role in evolutionary diversification, ant mimicry (myrmecomorphy) has evolved in at least 43 spider genera within 13 families [5].

The 300 or so species of described myrmecomorphic spiders are typically characterised by a thin, elongated body, the creation of an antennal "illusion" by waving the forelegs, and an erratic style of locomotion [5, 7-9]. The vast majority of these species are Batesian mimics that are avoided by antaverse arthropod predators [9-14], although the response of vertebrates is largely unknown. A few rough numbers may best express the efficacy of this deceptive signal. With over 5,300 described species, the Salticidae is the largest family of spiders [15]. The most speciose genus within the Salticidae, Myrmarachne, has over 200 described species_-all of them ant mimics.

Theoretically the Batesian mimics are under selective pressure to closely resemble their models while the models are under pressure to distance themselves from the deceitful signalling of the mimics, so there should be an arms race in which mimics are expected to converge upon their models (e.g., [16]). Yet polymorphism can also be maintained in populations of the Batesian mimics [17], particularly when more than one model species is available [3]. It is especially noticeable that several species of ant mimics are polymorphic $[18,19]$. As judged by humans, there is also considerable range in the accuracy of ant mimicry, with some being imprecise mimics, while others are remarkably similar in appearance to their model. Additionally, species in the large salticid genus Myrmarachne are sexually dimorphic as adults [20], with males seeming to be rather poor mimics due to their greatly enlarged chelicerae. Nevertheless, previous findings have suggested that males actually resemble ants carrying something in their mandibles [21]. In other words, they appear to be the Batesian mimics of a compound model (an ant plus the object it is carrying).

The exceptionally acute visual ability of salticids [22] enables them to identify motionless lures made from dead prey [23] and also enables them to escape some interactions with predators [11], such as ants. Although Myrmarachne can distinguish conspecifics and other mimics from ants [2426], current evidence suggests that non-ant-like salticids are unable to make this distinction $[4,21]$. The question of interest in this study is whether accuracy of ant mimicry, as judged by humans, is reflected in predator behaviour. The answer is of significance because most salticids will readily prey on each other [27], yet most salticids also appear to avoid ants [4], encounters with which are often lethal to salticids, including Myrmarachne [28, 29]. Clearly, it is also pertinent to determine how nonhuman animals classify objects and to determine the differences (or not) that may be found according to very different visual systems.

Here I tested Portia fimbriata, an Australian spider-eating (araneophagic) salticid that specialises on capturing other salticids as prey [30], with Asian weaver ants (Oecophylla smaragdina). I then compared whether their response toward ant-like salticids was similar to that elicited by O. smaragdina by testing $P$. fimbriata with males and females of Myrmarachne bakeri from the Philippines. This species is an imprecise ant mimic [19], and males are expected to be less precise than females due to their enlarged chelicerae. Finally, I tested $P$. fimbriata with an unrelated, but accurate, ant-like salticid from North America, Synageles occidentalis. In this study I address two specific questions: (1) does the non-antlike salticid $P$. fimbriata avoid ants? (2) does $P$. fimbriata avoid or stalk ant-like salticids, and does this predators' behaviour differ depending on the accuracy of the mimic?

\section{Materials and Methods}

I collected Myrmarachne bakeri and Oecophylla smaragdina in the Philippines and conducted laboratory work at the University of Canterbury (Christchurch, New Zealand), where cultures of Australian Portia fimbriata and North American Synageles occidentalis were available. Sexually mature female Portia fimbriata (body length $8-10 \mathrm{~mm}$ ) were tested with one of each of a variety of lures of four different types $(N=15$ for each type), and the distance to which $P$. fimbriata approached lures was measured. Lures were made from dead ants (major workers of $O$. smaragdina, $8 \mathrm{~mm}$ in body length) and ant mimics (male and female M. bakeri, 8 and $6 \mathrm{~mm}$ in body length, respectively, and female $S$. occidentalis, $3.5 \mathrm{~mm}$ in body length). While $M$. assimilis is the accurate mimic of $O$. smaragdina [4], there were no longer any individuals of this species in the laboratory in New Zealand when this study was done. As we were unable to procure any more, tests were carried out using another excellent mimic, S. occidentalis, instead. No test spiders had any previous experience with ants or with ant mimics.

Spiders were maintained in individual plastic cages, cleaned weekly, with a cotton roll through the bottom that dangled in a small cup of water to provide humidity. Spiders were fed twice a week with house flies (Musca domestica). Testing was done between $0800 \mathrm{~h}$ and $1700 \mathrm{~h}$ (laboratory photoperiod 12L:12D, lights on at $0800 \mathrm{~h}$ ). A $200 \mathrm{~W}$ incandescent lamp, positioned ca. $600 \mathrm{~mm}$ overhead, lit the apparatus; fluorescent lamps provided additional, ambient lighting. Using standard protocol for experiments on predatory behaviour, spiders were fasted between 4 to 7 days prior to testing. No individual spider was tested more than once with a given type of lure.

The testing apparatus was a wooden ramp (see Figure 1 for dimensions) raised at a $20^{\circ}$ angle, which was supported by a wooden pole, glued to a wooden base. The entire apparatus was painted with two coats of polyurethane and was wiped with $80 \%$ ethanol and allowed to dry for $30 \mathrm{~min}$ between each test to eliminate possible chemical traces from salticids in previous tests. The ramp was marked in a $5 \mathrm{~mm}$ grid to allow accurate distance measurements to be obtained. A thin piece of wood glued to the top end of the ramp served as a background against which the salticid saw the lure. The lure was placed $40 \mathrm{~mm}$ from the top end of the ramp, equidistant from both edges, and placed such that it was faced $45^{\circ}$ away from the pit, enabling test spiders to view cues from both the body and the head or cephalothorax of the lure. Lures were made by immobilizing an arthropod with $\mathrm{CO}_{2}$ and placing it in $80 \%$ ethanol. One day later, I mounted the arthropod in a life-like posture on the centre 


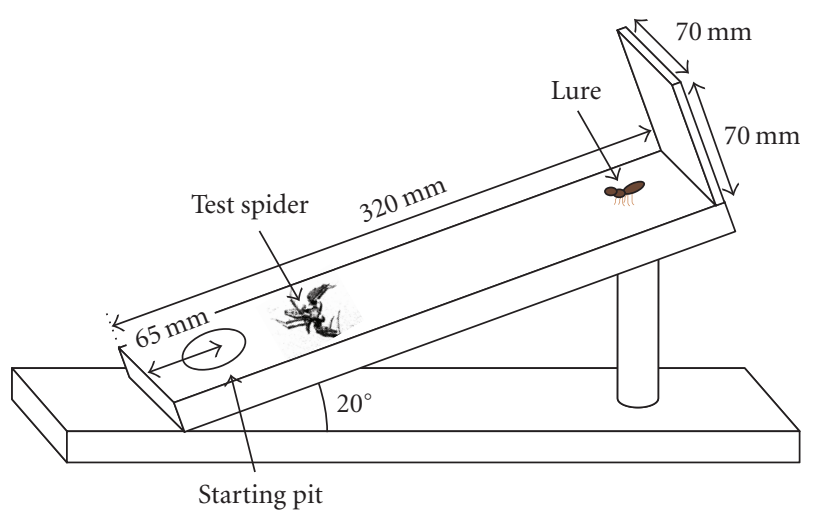

Figure 1: Ramp used for testing Portia fimbriata with lures of ants and ant mimics.

of one side of a disc-shaped piece of cork (diameter $c$. $1.25 \times$ the body length of the arthropod; thickness $c a$. $2 \mathrm{~mm}$ ) using forceps to position the arthropod. Lures were then sprayed with a transparent aerosol plastic adhesive for preservation.

Before each test, P. fimbriata was placed in a $32 \mathrm{~mm}$ diameter "starting pit" drilled halfway through the thickness of the ramp $200 \mathrm{~mm}$ from the lure. The salticid was left in the pit to acclimate for $60 \mathrm{~s}$ before a piece of cardboard, which was placed over the pit, was removed, allowing the salticid to exit from the pit. A white paper screen running along three sides surrounded the apparatus, leaving one side open for observations. The ramp was positioned so that the salticid moved away from the observer during tests. Tests began when $P$. fimbriata walked out of the pit and on to the ramp and ended when $P$. fimbriata either attacked the lure or walked off the top end of the ramp. If the salticid jumped off the ramp at a point below the lure or if it stayed in the pit for more than $30 \mathrm{~min}$ (no spiders walked under the ramp), tests were aborted. After testing for normality (D'Agostino and Pearson omnibus test), data were analysed using ANOVA in Prism v.5.

\section{Results}

There was a significant overall effect of lure type on the distance to which $P$. fimbriata approached the lure $\left(F_{3}=\right.$ 2.794, $P<0.05$ ), although in general $P$. fimbriata showed an aversion to both ants and ant mimics. P. fimbriata avoided contact with lures by circling around the lure and then continuing up the ramp. Tukey's post hoc comparisons revealed no differences between responses to $O$. smaragdina and S. occidentalis or female M. bakeri, but male M. bakeri were approached significantly closer than $O$. smaragdina $(P<$ $0.05)$. Overall $P$. fimbriata was kept furthest away from the ant (O. smaragdina), followed by $S$. occidentalis, then female M. bakeri, and lastly male M. bakeri (Figure 2). There were three instances of attacks towards lures, and all of these were aimed at lures of male M. bakeri.

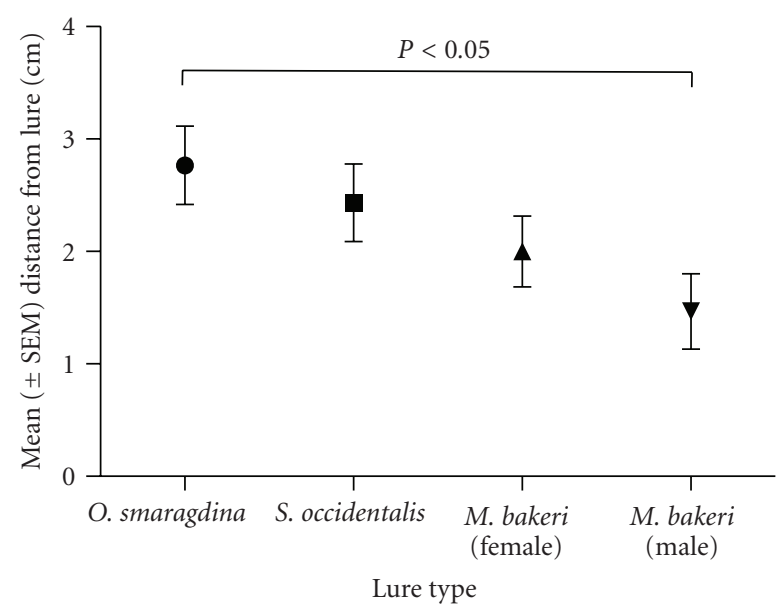

FIGURE 2: Mean $( \pm$ SEM) approach distance by the spider-eating salticid Portia fimbriata to lures of ants (Oecophylla smaragdina) and ant mimics of varying degrees of accuracy of mimicry (Synageles occidentalis and male and female Myrmarachne bakeri).

\section{Discussion}

Portia fimbriata was unable to correctly classify the mimics as its preferred prey, salticids [30], and instead generally responded toward the mimics as it did toward ants. These results provide additional evidence that ant mimicry in spiders functions as Batesian mimicry, even with naïve predators. However, it appears that the degree of resemblance to ants may have repercussions when faced with predators with acute eyesight, such as salticids. Synageles occidentalis is thought to mimic Lasius alienus or Myrmica americana, with which it is associated [10]. The salticids we had in the laboratory bore an extremely accurate resemblance to the former ant species. Although Myrmarachne bakeri resemble ants, they do not have a specific model to which they render a faithful portrait [19]. Portia fimbriata apparently also classified the potential prey with which it was faced in a similar manner to the way in which humans classify these animals, which is by no means a given. Males of M. bakeri were significantly less effective at deterring $P$. fimbriata than ants and slightly less aversive than M. bakeri females and $S$. occidentalis. Nevertheless, it should be noted that in these experiments prey behaviour was not taken into account. It is known, for example, that some myrmecomorphs will actively display to ant-eating salticid predators, deterring potential attack through mistaken identity [31]. While there is currently no evidence supporting the idea that accurate ant-like spiders behave more like ants than poor mimics, it is conceivable that this might have exacerbated the results of the current study.

The only striking visible difference between the male and the other stimulus animals was the male's large chelicerae. The chelicerae of sexually mature Myrmarachne males, which can increase their body size by $30-50 \%$ [27], is believed to have evolved as a sexually selected trait [32]. To our eyes, Myrmarachne males resemble ants considerably less convincingly than Myrmarachne females and juveniles, suggesting 
that, along with impaired feeding mechanics [32], impaired predator deterrence through inaccurate mimicry has been a cost of sexual dimorphism for male Myrmarachne. Contrary to the other potential prey, lures of male M. bakeri were occasionally attacked. Nevertheless, $P$. fimbriata generally avoided lures of male $M$. bakeri, suggesting that mimicry among males, despite possessing some cost in terms of diminished efficacy of mimicry due to their enlarged chelicerae, is still effective at deterring visually based predators. This supports the idea that the shape of the chelicerae of male Myrmarachne is in keeping with its mimicry because it looks like an ant worker carrying something in its mandibles [21], as is commonly observed in worker ants [6].

In a study using hoverfly mimics of wasps as prey and pigeons as predators, Dittrich et al. [33] found that despite some species being poor mimics, they were still protected by their mimicry, perhaps due to some constraint in the birds' visual or learning systems. Here it is apparent that imprecise mimics, although not avoided to the same degree as accurate mimics, were nevertheless aversive to naïve predators, suggesting that learning is not essential for the same effects to be seen. A mutually compatible alternative explanation is simply that very numerous and very dangerous models may produce a wider "cone of protection," thus allowing for imprecise mimicry [34] because the payoff to a predator for attacking prey with a given resemblance to a numerous and highly noxious model is limited [35]. Furthermore, polymorphic mimics that do not resemble any particular ant species especially closely may gain other advantages. For example, imprecise ant mimics may not be restricted to the geographical area or microhabitat (e.g., arboreal ants) in which a specific model species is found. Ants are notorious for both their abundance and their formidable defences [6], and it may not be surprising to find that among ant mimics there is considerable variation in form, ranging from accurate to imprecise mimicry. What is unusual is that here we have an example of a mimic resembling one of its own predators $[28,29]$.

\section{Acknowledgments}

Work in the Philippines was generously assisted by the International Rice Research Institute. Robert Jackson provided helpful suggestions on early versions of the manuscript.

\section{References}

[1] M. E. Edmunds, Defence in Animals: A Survey of Anti-Predator Defences, Longman, London, UK, 1974.

[2] G. D. Ruxton, T. N. Sherratt, and M. P. Speed, Avoiding Attack: The Evolutionary Ecology of Crypsis, Warning Signals and Mimicry, Oxford University Press, Oxford, UK, 2004.

[3] C. R. Darst and M. E. Cummings, "Predator learning favours mimicry of a less-toxic model in poison frogs," Nature, vol. 440, no. 7081, pp. 208-211, 2006.

[4] X. J. Nelson and R. R. Jackson, "Vision-based innate aversion to ants and ant mimics," Behavioral Ecology, vol. 17, no. 4, pp. 676-681, 2006.
[5] P. E. Cushing, "Myrmecomorphy and myrmecophily in spiders: a review," Florida Entomologist, vol. 80, no. 2, pp. 165193, 1997.

[6] B. Hölldobler and E. O. Wilson, The Ants, Springer, Heidelberg, Germany, 1990.

[7] J. Reiskind, "Ant-mimicry in Panamanian clubionid and salticid spiders (Araneae- Clubionidae, Salticidae)," Biotropica, vol. 9, pp. 1-8, 1977.

[8] P. S. Oliveira, "Ant-mimicry in some Brazilian salticid and clubionid spiders (Araneae: Salticidae, Clubionidae)," Biological Journal of the Linnean Society, vol. 33, no. 1, pp. 1-15, 1988.

[9] F. S. Ceccarelli, "Behavioral mimicry in Myrmarachne species (Araneae, Salticidae) from North Queensland, Australia," Journal of Arachnology, vol. 36, no. 2, pp. 344-351, 2008.

[10] B. Cutler, "Reduced predation on the antlike jumping spider Synageles occidentalis (Araneae: Salticidae)," Journal of Insect Behavior, vol. 4, no. 3, pp. 401-407, 1991.

[11] M. Edmunds, "Does mimicry of ants reduce predation by wasps on salticid spiders?" Memoires of the Queensland Museum, vol. 33, no. 2, pp. 507-512, 1993.

[12] M. Edmunds, "Do Malaysian Myrmarachne associate with particular species of ant?" Biological Journal of the Linnean Society, vol. 88, no. 4, pp. 645-653, 2006.

[13] X. J. Nelson and R. R. Jackson, "Collective Batesian mimicry of ant groups by aggregating spiders," Animal Behaviour, vol. 78, no. 1, pp. 123-129, 2009.

[14] X. J. Nelson, R. R. Jackson, D. Li, A. T. Barrion, and G. B. Edwards, "Innate aversion to ants (Hymenoptera: Formicidae) and ant mimics: experimental findings from mantises (Mantodea)," Biological Journal of the Linnean Society, vol. 88, no. 1, pp. 23-32, 2006.

[15] N. I. Platnick, “The world spider catalogue v.11.5,” 2010, http://research.amnh.org/iz/spiders/catalog/INTRO1.html.

[16] J. Mappes and R. V. Alatalo, "Batesian mimicry and signal accuracy," Evolution, vol. 51, no. 6, pp. 2050-2053, 1997.

[17] M. P. Speed and G. D. Ruxton, "Imperfect Batesian mimicry and the conspicuousness costs of mimetic resemblance," American Naturalist, vol. 176, no. 1, pp. E1-E14, 2010.

[18] F. S. Ceccarelli and R. H. Crozier, "Dynamics of the evolution of Batesian mimicry: molecular phylogenetic analysis of antmimicking Myrmarachne (Araneae: Salticidae) species and their ant models," Journal of Evolutionary Biology, vol. 20, no. 1, pp. 286-295, 2007.

[19] X. J. Nelson, "Polymorphism in an ant mimicking jumping spider," Journal of Arachnology, vol. 38, no. 1, pp. 139-141, 2010.

[20] F. R. Wanless, "A revision of the spider genera Belippo and Myrmarachne (Araneae: Salticidae) in the Ethiopian region," Bulletin of the British Museum of Natural History, vol. 33, pp. $1-139,1978$.

[21] X. J. Nelson and R. R. Jackson, "Compound mimicry and trading predators by the males of sexually dimorphic Batesian mimics," Proceedings of the Royal Society B, vol. 273, no. 1584, pp. 367-372, 2006.

[22] M. Land, "The morphology and optics of spider eyes," in Neurobiology of Arachnids, F. G. Barth, Ed., pp. 53-78, Springer, New York, NY, USA, 1985.

[23] X. J. Nelson and R. R. Jackson, "Prey classification by an araneophagic ant-like jumping spider (Araneae: Salticidae)," Journal of Zoology, vol. 279, no. 2, pp. 173-179, 2009.

[24] X. J. Nelson and R. R. Jackson, "Vision-based ability of an antmimicking jumping spider to discriminate between models, conspecific individuals and prey," Insectes Sociaux, vol. 54, no. 1, pp. 1-4, 2007. 
[25] F. S. Ceccarelli, "Ant-mimicking spider, Myrmarachne species (Araneae: Salticidae), distinguishes its model, the green ant, Oecophylla smaragdina, from a sympatric Batesian $O$. smaragdina mimic, Riptortus serripes (Hemiptera:Alydidae)," Australian Journal of Zoology, vol. 57, no. 5, pp. 305-309, 2009.

[26] X. J. Nelson, "Visual cues used by ant-like jumping spiders to distinguish conspecifics from their models," Journal of Arachnology, vol. 38, no. 1, pp. 27-34, 2010.

[27] R. R. Jackson and S. D. Pollard, "Predatory behavior of jumping spiders," Annual Review of Entomology, vol. 41, no. 1, pp. 287-308, 1996.

[28] X. J. Nelson, R. R. Jackson, G. B. Edwards, and A. T. Barrion, "Living with the enemy: jumping spiders that mimic weaver ants," Journal of Arachnology, vol. 33, no. 3, pp. 813-819, 2005.

[29] X. J. Nelson, R. R. Jackson, S. D. Pollard, G. B. Edwards, and A. T. Barrion, "Predation by ants on jumping spiders (Araneae: Salticidae) in the Philippines," New Zealand Journal of Zoology, vol. 31, no. 1, pp. 45-56, 2004.

[30] D. P. Harland and R. R. Jackson, "Portia Perceptions: the umwelt of an araneophagic jumping spider," in Complex Worlds from Simpler Nervous Systems, F. R. Prete, Ed., pp. 540, MIT Press, Cambridge, Mass, USA, 2004.

[31] X. J. Nelson, R. R. Jackson, and D. Li, "Conditional use of honest signaling by a Batesian mimic," Behavioral Ecology, vol. 17, no. 4, pp. 575-580, 2006.

[32] S. D. Pollard, "Consequences of sexual selection on feeding in male jumping spiders (Araneae: Salticidae)," Journal of Zoology, vol. 234, no. 2, pp. 203-208, 1994.

[33] W. Dittrich, F. Gilbert, P. Green, P. Mcgregor, and D. Grewcock, "Imperfect mimicry: a pigeon's perspective," Proceedings of the Royal Society B, vol. 251, no. 1332, pp. 195-200, 1993.

[34] D. W. Kikuchi and D. W. Pfennig, "High-model abundance may permit the gradual evolution of Batesian mimicry: an experimental test," Proceedings of the Royal Society B, vol. 277, no. 1684, pp. 1041-1048, 2010.

[35] T. N. Sherratt, "The evolution of imperfect mimicry," Behavioral Ecology, vol. 13, no. 6, pp. 821-826, 2002. 

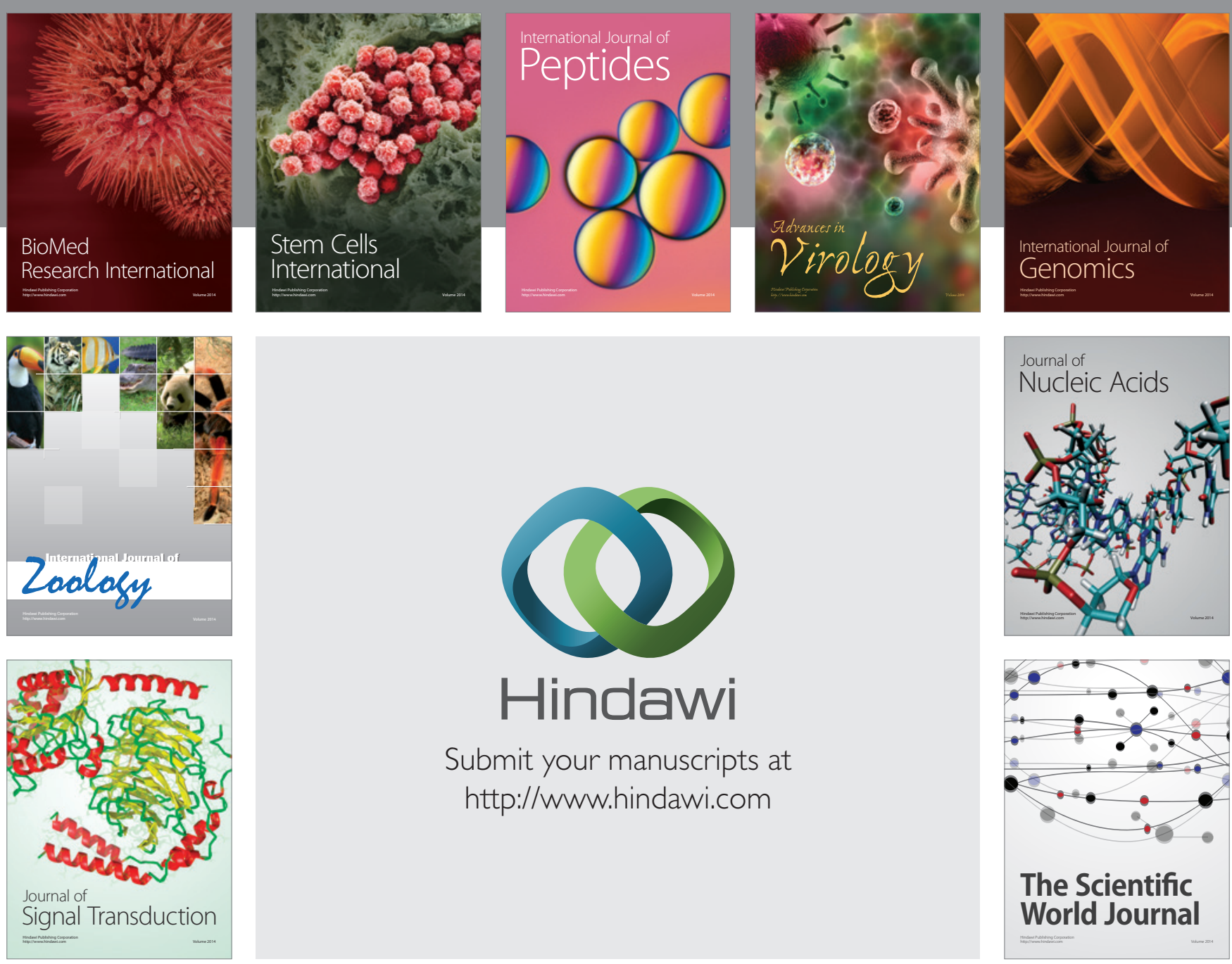

Submit your manuscripts at

http://www.hindawi.com
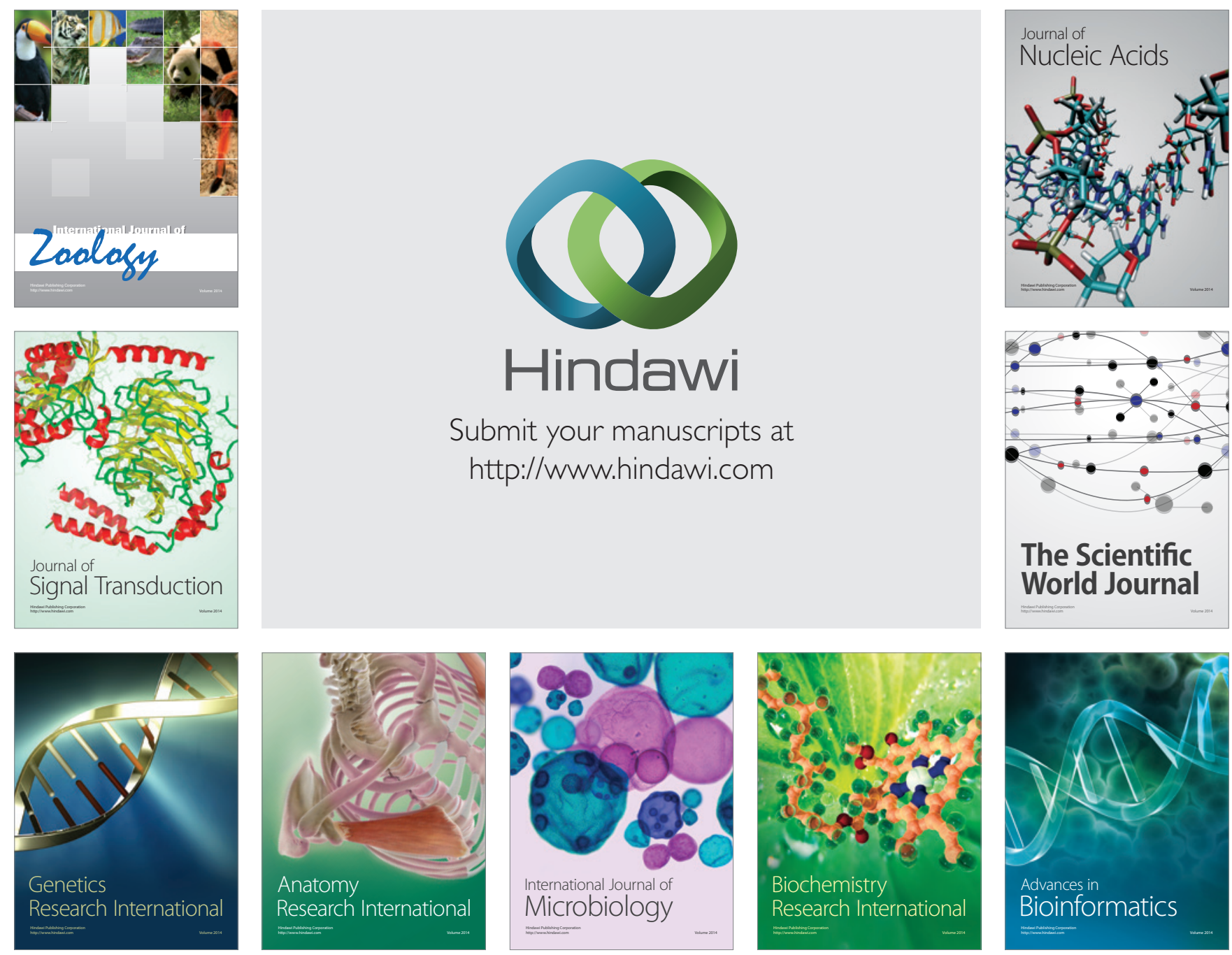

The Scientific World Journal
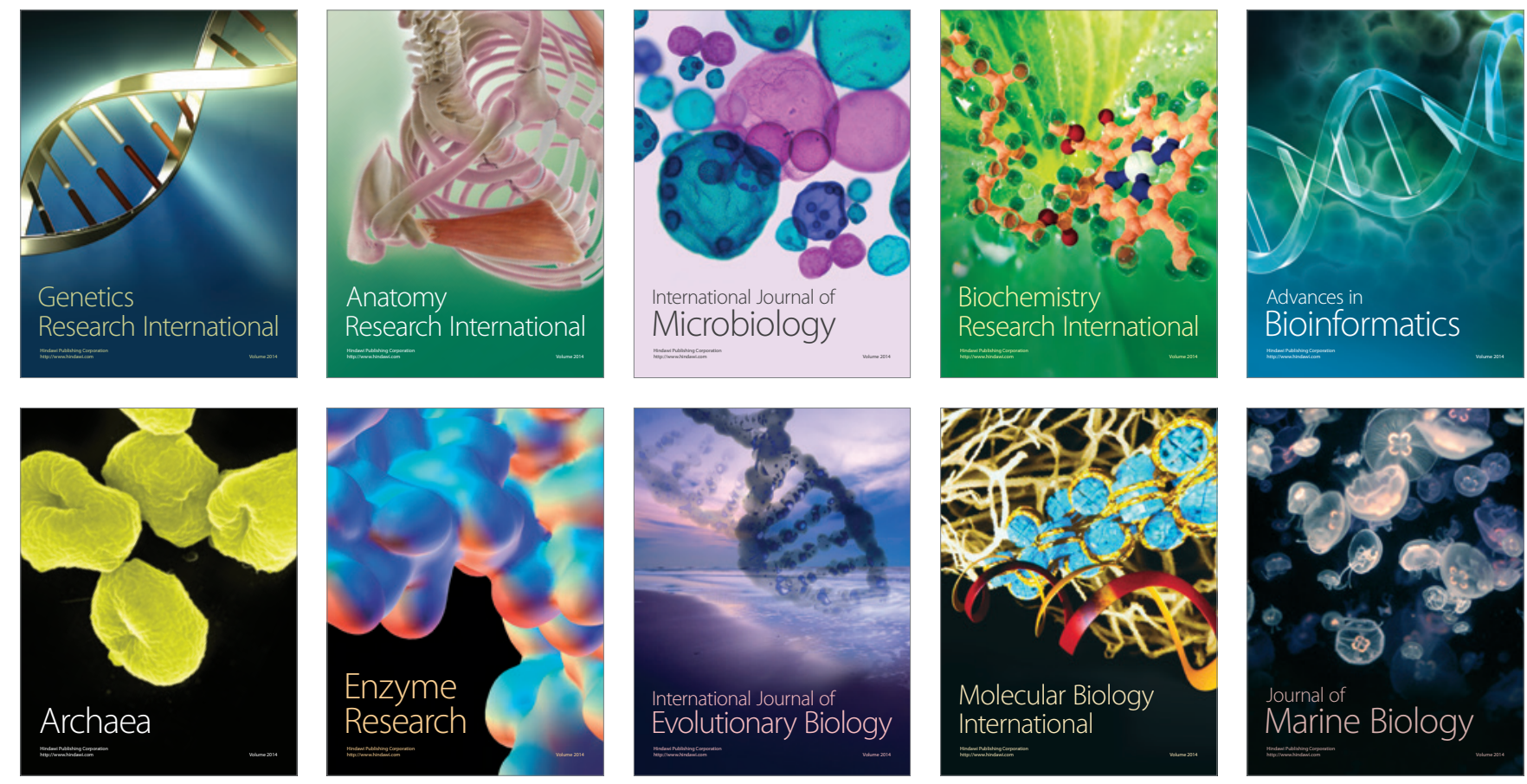